\title{
Recurrent pulsations in Saturn's high latitude magnetosphere
}

\author{
D.G. Mitchell ${ }^{\text {a,* }}$, J.F. Carbary ${ }^{\text {a }}$, E.J. Bunce ${ }^{\text {b }}$, A. Radioti ${ }^{c}$, S.V. Badman ${ }^{d}$, W.R. Pryor ${ }^{\mathrm{e}}$, G.B. Hospodarsky ${ }^{\mathrm{f}}$, \\ W.S. Kurth ${ }^{\mathrm{f}}$ \\ a Johns Hopkins University Applied Physics Laboratory, Laurel, MD 20723, USA \\ ${ }^{\mathrm{b}}$ Department of Physics and Astronomy, University of Leicester, Leicester, UK \\ ' Laboratoire de Physique Atmosphérique et Planétaire, Institut d'Astrophysique et de Géophysique, Université de Liège, Liege, Belgium \\ ${ }^{\mathrm{d}}$ Department of Physics, Lancaster University, Lancaster, UK \\ ${ }^{\text {e } C e n t r a l ~ A r i z o n a ~ C o l l e g e, ~ C o o l i d g e, ~ A Z ~ 85128, ~ U S A ~}$ \\ ${ }^{\mathrm{f}}$ Department of Physics and Astronomy, University of Iowa, Iowa City, IA 52242, USA
}

\section{A R T I C L E I N F O}

\section{Article history:}

Received 12 May 2014

Revised 17 September 2014

Accepted 16 October 2014

Available online $\mathrm{xxxx}$

\section{Keywords:}

Saturn, magnetosphere

Aurorae

Magnetospheres

Ionospheres

Solar wind

\begin{abstract}
A B S T R A C T
Over the course of about 6 h on Day 129, 2008, the UV imaging spectrograph (UVIS) on the Cassini spacecraft observed a repeated intensification and broadening of the high latitude auroral oval into the polar cap. This feature repeated at least 5 times with about a $1 \mathrm{~h}$ period, as it rotated in the direction of corotation, somewhat below the planetary rotation rate, such that it moved from noon to post-dusk, and from roughly $77^{\circ}$ to $82^{\circ}$ northern latitudes during the observing interval. The recurring UV observation was accompanied by pronounced $\sim 1 \mathrm{~h}$ pulsations in auroral hiss power, magnetic perturbations consistent with small-scale field aligned currents, and energetic ion conics and electrons beaming upward parallel to the local magnetic field at the spacecraft location. The magnetic field and particle events are in phase with the auroral hiss pulsation. This event, taken in the context of the more thoroughly documented auroral hiss and particle signatures (seen on many high latitude Cassini orbits), sheds light on the possible driving mechanisms, the most likely of which are magnetopause reconnection and/or Kelvin Helmholtz waves.
\end{abstract}

(c) 2014 Elsevier Inc. All rights reserved.

\section{Introduction}

Pulsations in auroral hiss as seen by the Cassini Radio and Plasma Wave Science (RPWS, Gurnett et al. (2004)) instrument aboard the Cassini spacecraft have been observed on many occasions, often in association with correlated energetic ion conics, high energy, field aligned electrons, and magnetic signatures of field aligned currents (Mitchell et al., 2009a). These events formed a pulsed subcategory of a broader class of events documented in that work, all of which were associated with downward field aligned currents (FAC). The broader class was thought to represent the Saturn equivalent of similar field-aligned electron beam events observed at Earth in association with downward field-aligned currents in the auroral zone (Klumpar, 1990; Carlson et al., 1998; Marklund et al., 2001), although this subclass of pulsed events has not been documented at Earth. Auroral features at high latitudes and local times (i.e., rotating from near noon toward dusk), similar to those found for the series of pulsations presented here, have also been observed on other occasions at Saturn. Radioti et al. (2011, 2013) and Badman et al. $(2012,2013)$ have showed observations

\footnotetext{
* Corresponding author.
}

of auroral bifurcations of the main emission, and related them to magnetopause reconnection. Radioti et al. (2013) showed two "rebrightenings" of the bifurcations of the main emissions, with an interval of $1 \mathrm{~h}$, but no other mention has been made of the repeated brightening at about a $1 \mathrm{~h}$ period in the auroral data. There has been no association made between these repeated auroral events and the $\sim 1 \mathrm{~h}$ pulsations observed regularly in the radio wave and particle data, for example in Mitchell et al. (2009a), Jasinski et al. (2014), Bunce et al. (2014). Badman et al. (2012) showed the correspondence of similar $1 \mathrm{~h}$ pulsations in the in situ data with the presence of conjugate auroral arcs, but did not have the temporal resolution to observe any intensity variations in the auroral emissions. It has been suggested by Radioti et al. (2011, 2013) and Badman et al. $(2012,2013)$ that repeated magnetopause reconnection may be responsible for the auroral features, where it has also been documented that they significantly subcorotate.

\section{Observations}

The UV auroral observations of Saturn's northern ionosphere begin just before 0800 UT on May 8 (day 129), 2008, and repeat 
at a cadence of four full auroral images per hour until just before 1400 UT. Fig. 1 shows a sequence of polar projections of Saturn's northern aurora obtained with the FUV channel (111-191 nm) of the Cassini UVIS instrument (Esposito et al., 2004) on DOY 129, 2008 , one hour per horizontal line of four images. Circles on each figure are drawn at $70^{\circ}$ and $80^{\circ}$ colatitude, and in white in the upper left panel we show the trajectory of the Cassini spacecraft mapped to the ionosphere, with the interval analyzed in the paper highlighted in pink and labeled with a pink " $\mathrm{C}$ ".

The images reveal a rotating region of bright arcs moving from near noon through dusk between northern co-latitudes of $\sim 8-13^{\circ}$, whose intensity varies considerably from image to image in a repeating fashion. These arcs are labeled with a white " $\mathrm{B}$ " in each frame for which their emission peaks. As in Radioti et al. (2011, 2013), and Badman et al. (2013) this region is closely associated with a strong distortion of the auroral oval into the polar cap just westward of the recurrent brightening. Also apparent, though much fainter, is a recurrent brightening near noon at high colatitudes between 3 and $12^{\circ}$, a region that may be connected with the cusp (Gérard et al., 2005; Bunce et al., 2005). The frames for which this noon, high latitude emission reaches peak intensity are labeled with the word "cusp?".

To quantify the intensity of the emissions associated with these auroral regions, in Fig. 2a and b we construct auroral keograms, as in Mitchell et al. (in press), by averaging the auroral emissions from each panel of Fig. 1 in local time bins of $1 \mathrm{~h}$ between colatitudes of $10-15^{\circ}$ (a) and $3-10^{\circ}$ (b). The former colatitude range captures essentially the entire poleward edge of the auroral oval (e.g., Badman et al., 2014), while the latter includes primarily the polar cap (containing the possible aforementioned cusp-related auroral emission near to noon). These ranges were chosen so as to isolate the "cusp?" signature from the pulsating bifurcated arc signature in the keograms. The top panels of Fig. 2 exhibit the local time keograms obtained the full set of auroral scans in Fig. 1. Also included in Fig. 2a is a blue line that tracks the local time of Saturn's moon, Mimas. The results obtained using this line instead of the white line that was drawn to follow the arcs are indistinguishable from those using the white line, corresponding to an orbital period of 23 h, i.e., approximately $50 \%$ of corotation. We have no mechanism to offer to explain this causally, but simply point it out as a curiosity. However, $50 \%$ of corotation is a fairly typical magnitude both in the polar auroral region (e.g., Nichols et al., 2014) and for plasma in the outer magnetosphere (Thomsen et al., 2010; Arridge et al., 2011).

Even a cursory inspection of the keograms reveals the time variations of the auroral regions of interest in this study. White lines have been drawn across the each of the keograms that roughly track the relevant auroral features. One can arrive at an estimate of the intensities of the features as a function of time by averaging the intensities, for each scan, within $\pm 2 \mathrm{~h}$ of the local times of these lines. These intensities-along-the-lines appear in the bottom panels of Fig. 2.

This event took place during the same time interval discussed in Mitchell et al. (2009b) [see their Figs. 5 and 7 containing MIMI/ INCA energetic neutral atom (ENA) images, along with the discussion]. We wish to emphasize that aside from a likely common association with a high pressure solar wind driver, we believe this event to be unrelated with the bright system of arcs beginning post-midnight and rotating through dawn during this same sequence, at somewhat lower latitudes (seen in Fig. 1, as well as in the morning local times in the $10-15^{\circ}$ keogram in Fig. 2). That region was discussed in some detail in Mitchell et al. (2009b), and related to probable nightside reconnection and subsequent ring current heating and rotation as imaged in energetic neutral atoms. There was no enhanced ENA emission associated with this pulsating auroral activity, consistent with the high latitude of the auroral features, which most likely map to the dusk side magnetopause, a region where energetic ion intensities are not generally high enough to produce ENA emission above the INCA threshold for detection.

The in situ measurements at Cassini (Fig. 3), however, show very strong correlation with the auroral activity. During the interval shown in Fig. 3, the spacecraft position mapped to the northern hemisphere high-latitude polar cap at approximately constant colatitude of $\sim 8^{\circ}$ (as shown by the pink line in Fig. 1). The spacecraft was located at high latitudes off the equator in the middle to outer magnetosphere (between radial distances of $\sim 15$ and $17 R_{\mathrm{S}}$ ), near to $13 \mathrm{~h} \mathrm{LT}$. The top panel (A) shows the magnetic field components from the Cassini magnetometer instrument (MAG, Dougherty et al. (2004)). The second panel (B) shows the magnetically mapped ionospheric footprint of Cassini relative to the model location of the nominal auroral, using a combination of the internal field model of Dougherty et al. (2005) and the ring current model of Bunce et al. (2007). In addition a model location of the nominal auroral oval at the longitude of the spacecraft is shown based on the northern magnetosphere oscillation phase for this interval (see Andrews et al. (2012)), and a model of the observed amplitude of the oscillation of the auroral oval by Nichols et al. (2010). The third panel (C) shows rates resulting from field aligned energetic electrons (discussed in the following paragraph), and the last panel (D) presents RPWS auroral hiss intensities.

In panel $C$ of Fig. 3, we plot the counting rate from the MIMI/ INCA "Stop" microchannel plate (MCP). Whereas INCA is designed to measure ENAs and ions, the MCP detectors used to derive the speed and direction of the ENAs or ions also respond with reasonable efficiency to energetic electrons. Because the "Start" MCP in INCA is not in the particle trajectory path, the signal from electrons in the Start MCP is weak-it relies on secondary electrons generated in the Start entrance foil as primary energetic particles transit the foil on their way through the time of flight (TOF) volume to the Stop foil and MCP. The primary particles penetrate the Stop foil directly in front of the Stop MCP, and collide with the Stop MCP. Although energetic electrons have a low efficiency for generating secondary electrons in the Start foil $(<1 \%)$, they have a high probability of triggering the Stop MCP (10s of percent). With INCA in ENA imaging mode (as it was throughout this event) only charged particles with $E / q \geqslant 180 \mathrm{keV} / \mathrm{q}$ can get through the high voltage charged particle rejection plates in front of the entrance slit. Of these, the efficiency for proton detection above that energy is roughly $30 \%$, for which we measure the proton TOF and so derive its energy and trajectory. Electrons not only have a low probability of generating a TOF because of the low Start efficiency for electrons, but their TOF is shorter $(\sim 1-2 \mathrm{~ns})$ than the minimum TOF analyzed by INCA, and we do not know their energy other than that it is sufficient to enter through the charged particle rejection plates. So the signature of energetic electrons in INCA is simply elevated Stop counting rates without a corresponding increase in Start counting rates. Additional detail on the detection techniques used in INCA measurements can be found in the MIMI instrument paper, Krimigis et al. (2004).

The energetic electron intensity is not steady, but beginning a little before 10:00 UT comes and goes episodically, with fast fluctuations modulated by an envelope with a period of about $1 \mathrm{~h}$. The auroral hiss power (bottom panel) likewise varies quasi-periodically, although the average power varies by about an order of magnitude over the interval between 0600 and 1600 UT. Indications from the MIMI CHEMS instrument are that magnetospheric ion intensities were slightly higher in the interval between 0800 and 1000 UT, consistent with the spacecraft being farther inside the magnetopause at this time than over the rest of the interval. The magnetic field behavior (panel A) is less obviously periodic, but the phi and theta angles vary consistent with Alfvénic structures carrying field-aligned 


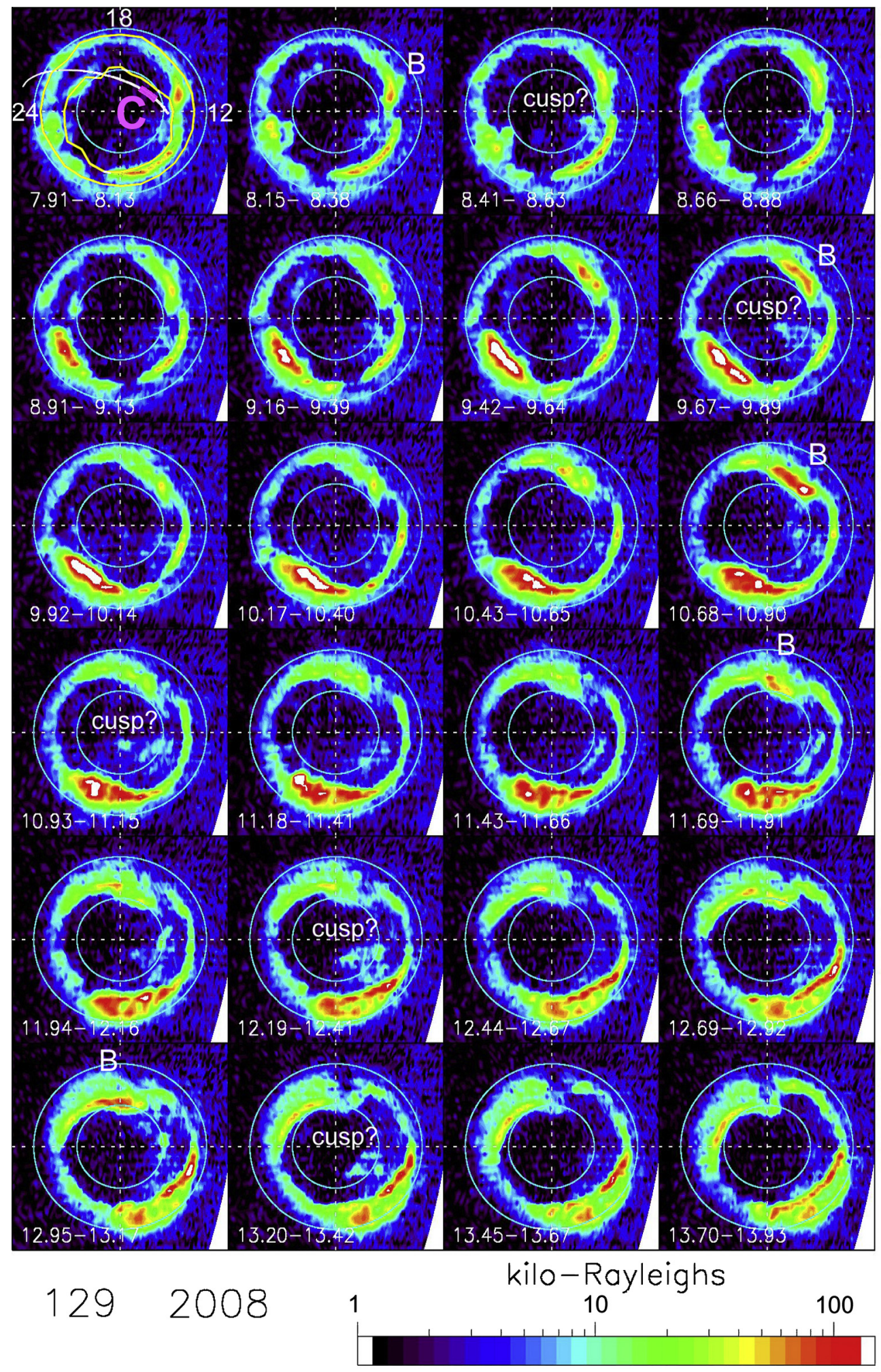

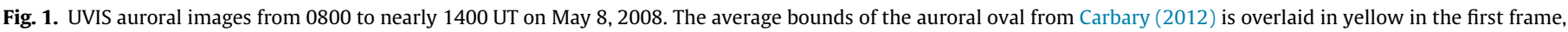
and the magnetically mapped spacecraft trajectory using a combination of the internal field model of Dougherty et al. (2005) and the ring current model of Bunce et al. (2007) is shown in white. The observation period discussed in this paper (labeled " $\mathrm{C}$ " for Cassini) is highlighted in pink. Image acquisition times in UT appear on each frame. Circles are drawn at $10^{\circ}$ and $20^{\circ}$ colatitude, and the Sun is to the right, with 12,18, and 24 LT labeled in the first frame. The view is from the north pole looking down the spin axis of Saturn. The images have been corrected for aspect and wavelength filtering. The pulsating bright arc is labeled with a white "B" at the peak of each pulsation. The word "cusp?" appears in those frames for which the polar cap/cusp region emission peaked. The bright emission at lower latitude on the dawn side is associated with magnetotail activity, and is not though to be associated with the pulsations discussed here. 

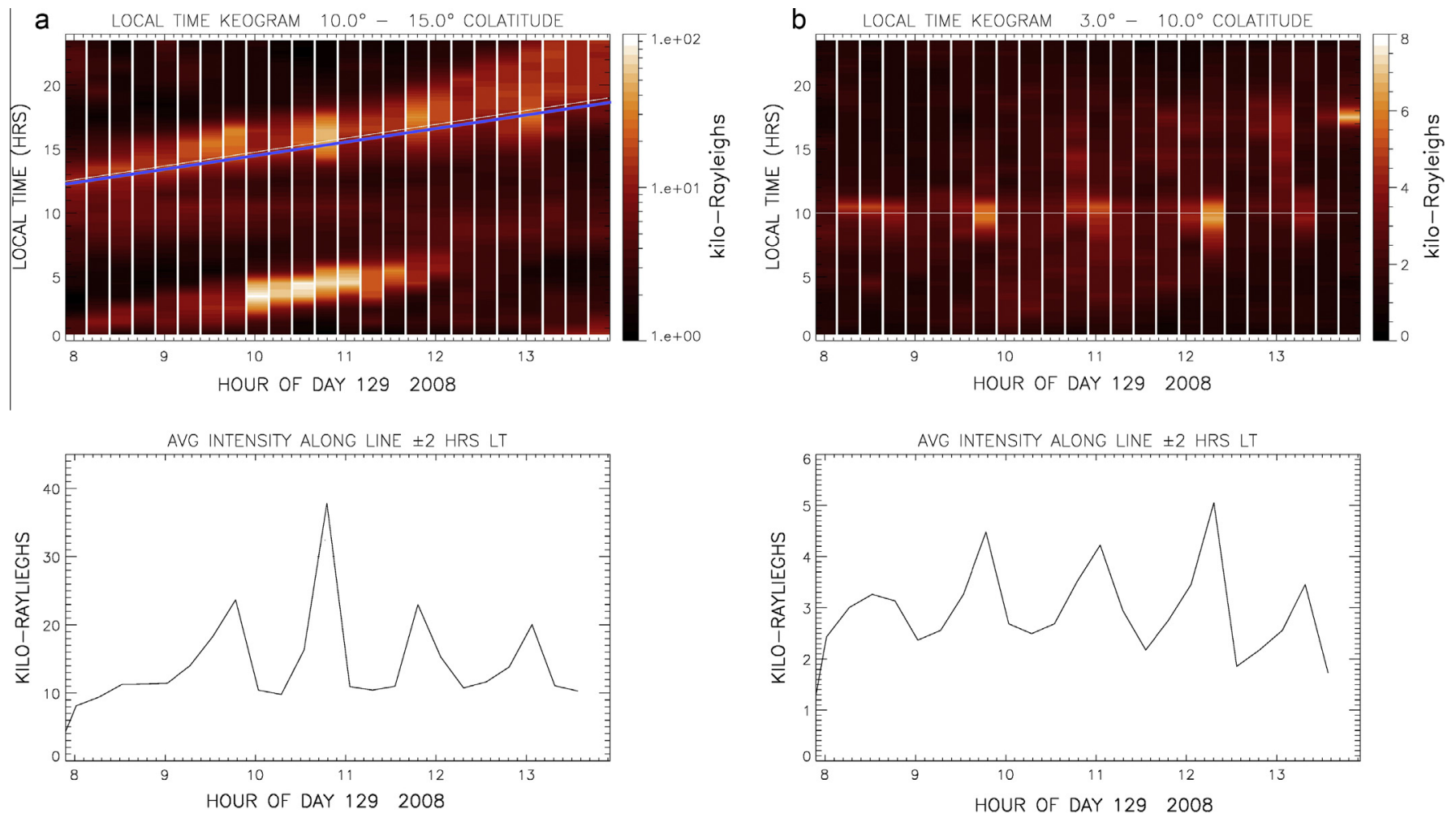

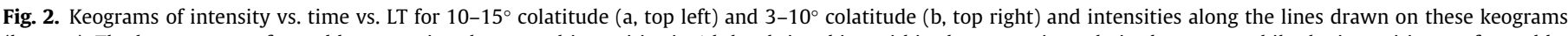

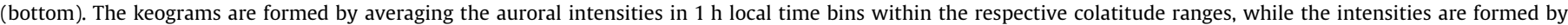

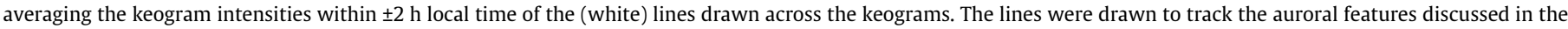
text. For the lower latitude keogram (a) a blue line follows the local time location of Saturn's moon Mimas.

current passing over the spacecraft, and those fluctuations are correlated with the quasi-periodic variation of the electron and auroral hiss intensities. In Fig. 3B, which shows the model colatitude of Cassini's ionospheric footprint (black line), we see that Cassini was located on field lines mapping significantly poleward of the typical (and observed) auroral oval during this interval.

To explore the relationship between these measurements more directly, we further process the data by detrending and rescaling them so that the modulations in each data set can be compared for phase. Fig. 4 shows the relationship between these adjusted pulsations seen in the various fields, particles and auroral emissions. The auroral hiss is generated along the auroral field lines by the streaming electrons that carry the downward field aligned current. Its integrated, averaged power (Fig. 3, black line) has been de-trended and re-scaled so that the $\sim 1 \mathrm{~h}$ modulation can be easily compared with the other data products. The INCA electron response has been averaged with a 10-min running box-car average to remove the fine structure. The auroral hiss and the energetic electrons are modulated approximately in phase with each other. Likewise, the high latitude polar cap emission ("cusp?") brightens and dims in phase with the in situ data. The correlation is very clear, implying a rather direct correspondence between the high altitude in situ plasma and field measurements, and the high latitude auroral emission pulsation in the northern ionosphere. The bifurcated arc, on the other hand, is modulated at very nearly the same period, but leads the other quantities by $\sim 18 \mathrm{~min}$.

\section{Discussion/conclusions}

Based on the high latitude, as well as the absence of associated emission in ENA from the magnetosphere, this auroral phenome- non is associated with field lines connected to the outer magnetosphere very close to the magnetopause, and/or in the cusp/mantle near to $13 \mathrm{~h} \mathrm{LT}$.

This pulsation is likely driven by field-aligned currents associated with flow shears in the solar wind-magnetopause-low latitude boundary layer and/or mantle interface. These field-aligned currents are small in scale relative to the large-scale field-aligned current structures associated with the main auroral oval as described for example in Bunce et al. (2008). Nevertheless, a large region of the auroral zone and polar cap is apparently involved (see below). The pulsation is consistent with recurrent reconnection (e.g., Radioti et al., 2011; Badman et al., 2013) and/or possibly with Kelvin-Helmholtz wave activity along the dusk flank, where $\mathrm{KH}$ waves have been well documented (Masters et al., 2010; Wilson et al., 2012; Delamere et al., 2013). The auroral brightening in the cusp region may favor a reconnection interpretation (e.g., Meredith et al., 2014).

The observed particles and waves are consistent with similar observations discussed in Mitchell et al. (2009a) that have previously been associated with auroral activity on both open and closed field line configurations (as determined by energetic electron anisotropies). The pulsations seen in the in situ data are similar to those discussed in Mitchell et al. (2009a), Badman et al. (2012), Jasinski et al. (2014), and Bunce et al. (2014), although in this event we do not have the required data to make a direct inference regarding the field topology.

The brightest auroral pulsation, likely a bifurcated arc structure, rotates through $\sim 6 \mathrm{~h}$ of local time (from noon to past dusk) over the course of the observations, suggesting a persistent structure of shear flow and field-aligned current carried anti-sunward along the dusk flank, presumably at or near to the magnetopause. 

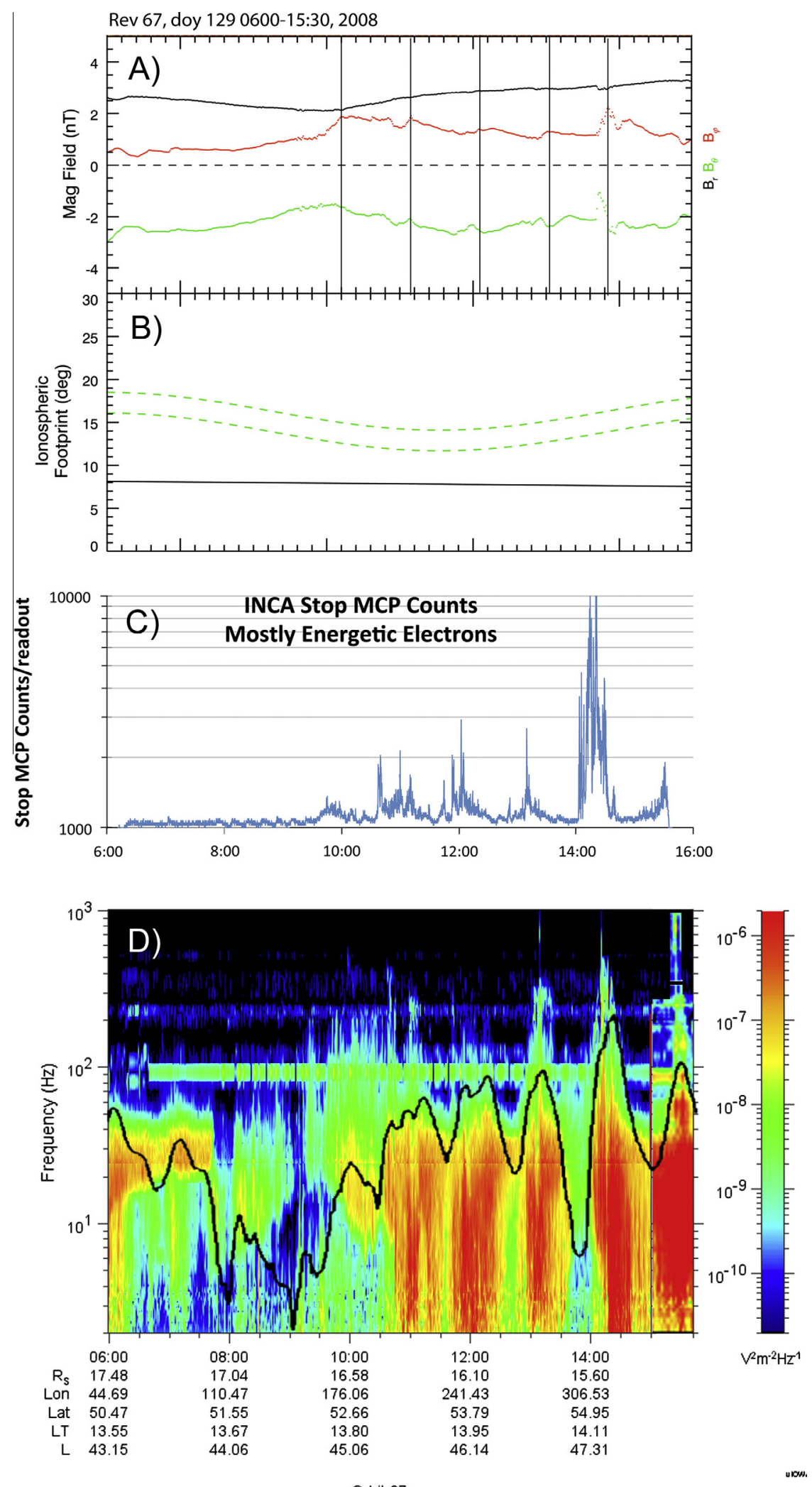

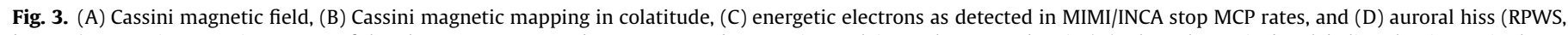

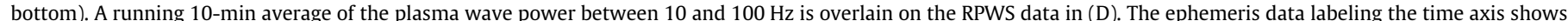

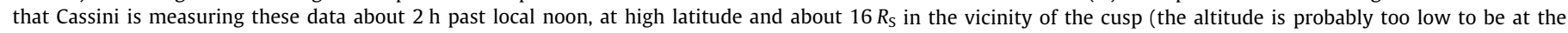
magnetopause).

The strongest energetic electron signature and the strongest small-scale field-aligned current signature are both in phase with the most intense auroral hiss observed (at $1400 \mathrm{LT}, 15.6 R_{\mathrm{S}}$ and $55^{\circ}$ latitude), suggesting that the spacecraft was close to or on the magnetic field lines connecting to the auroral pulsations. This observation occurs as the brightest portion of the rotating auroral 


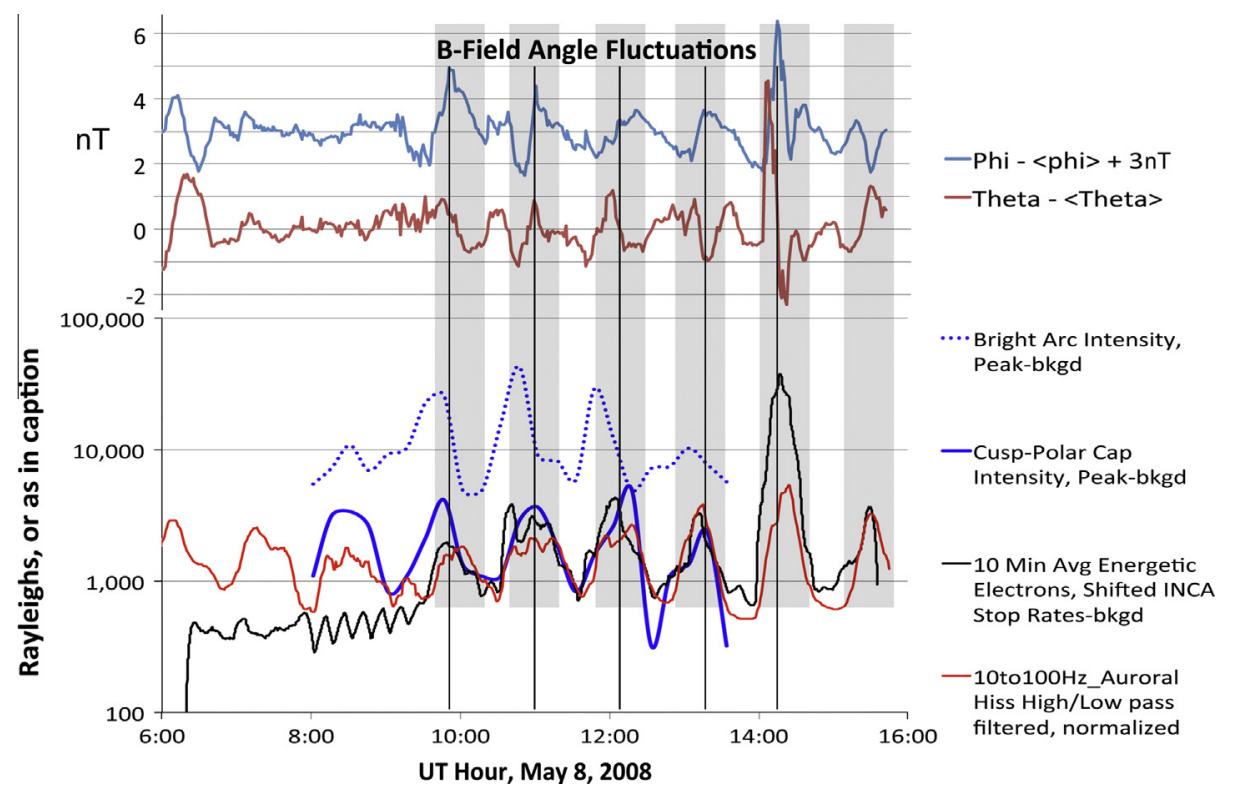

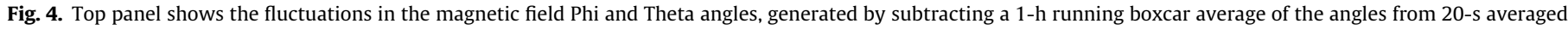

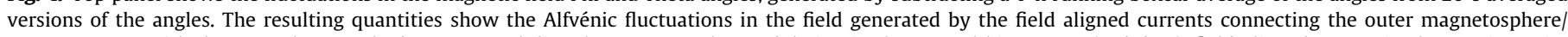

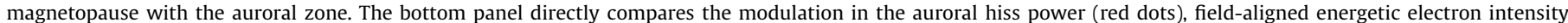

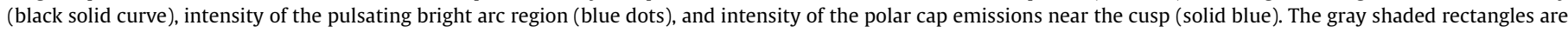

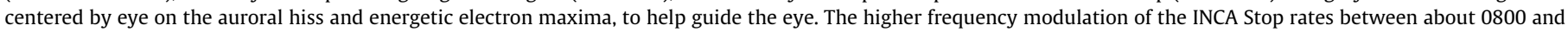
0930 is caused by spacecraft attitude changes moving the sensor relative to an electron beam, and is not directly related to the environment.

arc reaches approximately 2000 LT. A direct association between the in situ observations at 14:00 h LT and the brightest auroral arc emission at 20:00 h LT would therefore require a significant (and implausible) distortion of the magnetic field between high and low altitude, and is further complicated by the $\sim 18$ min phase lag of the pulsations of the in situ data relative to the pulsations of the bright auroral arc.

An alternative to the requirement to reconcile the incorrect mapping between the bright auroral arc and the in situ observations is that the currents, particles and auroral hiss emissions are actually connected to cusp and/or polar cap field lines, and not magnetically connected to the most intense auroral arc brightening taking place between noon and dusk (and spanning several hours of local time). The in situ plasma observations are indicative of temporal variation of a local downward current region, while the auroral brightenings are likely related to electron precipitation in adjacent upward current regions. The cusp modeling of Bunce et al. (2005) provide estimates of the cusp-related plasma flows and associated field-aligned current morphologies for a variety of interplanetary magnetic field (IMF) conditions. The latitude (i.e., along the main oval or poleward of it), longitude (i.e., East or West end of the merging gap) and brightness of the features depend on a combination of IMF $B_{\mathrm{z}}$ and $B_{\mathrm{y}}$ conditions. Their model assumed that the main oval (or upward-directed field-aligned current) was co-located with the open-closed field line boundary, which we now know is not the case (Talboys et al., 2011; Jinks et al., 2014), and hence any features proposed by Bunce et al. (2005) to lie along the main oval, may instead lie poleward of it by a few degrees co-latitude. Talboys et al. (2011) and Jinks et al. (2014) showed that the upward fieldaligned current associated with the main auroral oval on the nightside lies $1-2^{\circ}$ (is this the right value?) equatorward of the open-closed field line boundary determined from particle observations. Modeling and observations of the dawn side aurorae suggest they map to the outer ring current, while the noon and post-noon aurorae display various features including 'cusp' features at or poleward of the open-closed boundary, and poleward bifurcations believed to map to newly-open field lines (Meredith et al., 2014).

However, given that we do not know the direction of the IMF throughout this interval we cannot interpret the precise details of the cusp-like morphologies seen here. As already discussed, we do know that the observations discussed here may have occurred during a solar wind compression event and it thus seems plausible that the fainter emissions at high latitude near to noon are associated with enhanced dayside reconnection efficiency during such disturbed solar wind conditions. Bunce et al. (2005) also suggest that such reconnection may be characteristically "pulsed" with a time-scale of $\sim 30 \mathrm{~min}$ (based on the Alfven travel time on the last closed field line). This may offer an explanation for the pulsing of the noon cusp emission. Of course, how this links to the physical origin and approximately fixed phase relation of the pulsing of the bright auroral arc between noon and dusk remains an open question here.

Given their nearly identical periods, we believe the behavior of the in situ and cusp regions to be causally related to the bright auroral pulsation implying the involvement of a very large region of the high latitude ionosphere. The bright auroral pulsation leads the cusp pulsation by $\sim 18 \mathrm{~min}$, which may point to the existence of a pressure pulse or wave traveling across the polar cap in the open field region away from the poleward boundary of the auroral arc emission. Auroral flares covering large spatial regions have previously been identified at Jupiter and related to magnetopause processes, which could be analogous to those identified in this study (Bonfond et al., GRL, 2011).

This auroral emission has many similarities to events discussed in the literature previously (Radioti et al., 2013; Badman et al., 2013 ; etc.), but has some important additional features: the rotation of the bright arcs through more than $6 \mathrm{~h}$ of local time from noon through to dusk, and the clear involvement of a large fraction of the polar cap, including a correlated periodic intensity enhancement the high latitude cusp region. 


\section{Acknowledgments}

This research was supported by the NASA Office of Space Science under Task Order 003 of contract NAS5-97271 between NASA Goddard Space flight Center and the Johns Hopkins University. EJB is supported by the STFC Leicester Consolidated Grant ST/K001000/1, and by a Philip Leverhulme Award. The research at the University of Iowa was supported by NASA through Contract 1415150 with the Jet Propulsion Laboratory. SVB is supported by a Royal Astronomical Society Research Fellowship.

\section{References}

Andrews, D.J., Cowley, S.W.H., Dougherty, M.K., Lamy, L., Provan, G., Southwood, D.J., 2012. Planetary period oscillations in Saturn's magnetosphere: Evolution of magnetic oscillation properties from southern summer to post-equinox. J. Geophys. Res. 117, A04224. http://dx.doi.org/10.1029/2011JA017444.

Arridge et al., 2011. Mapping Magnetospheric Equatorial Regions at Saturn from Cassini Prime Mission Observations. (ISSI) Space Sci. Rev. 164, 1-83. http:// dx.doi.org/10.1007/s11214-011-9850-4.

Badman, S.V, et al., 2012. Cassini observations of ion and electron beams at Saturn and their relationship to infrared auroral arcs. J. Geophys. Res. 117, A01211. http://dx.doi.org/10.1029/2011JA017222.

Badman, S.V. et al., 2013. Bursty magnetic reconnection at Saturn's magnetopause. Geophys. Res. Lett. 40, 1027-1031. http://dx.doi.org/10.1002/grl.50199.

Badman, S.V. et al., 2014. Auroral processes at the giant planets: Energy deposition, emission mechanisms, morphology, and spectra. Space Sci. Rev. http:/ dx.doi.org/10.1007/s11214-014-0042-x.

Bonfond, B., Vogt, M.F., Gérard, J.-C., Grodent, D., Radioti, A., Coumans, V., 2011. Quasi-periodic polar flares at Jupiter: A signature of pulsed dayside reconnections? Geophys. Res. Lett. 38, L02104. http://dx.doi.org/10.1029/ 2010 GL045981.

Bunce, E.J., Cowley, S.W.H., Milan, S.E., 2005. Interplanetary magnetic field control of Saturn's polar cusp aurora. Ann. Geophys. 23, 1405-1431. http://dx.doi.org 10.5194/angeo-23-1405-2005.

Bunce, E.J. et al., 2007. Cassini observations of the variation of Saturn's ring current parameters with system size. J. Geophys. Res. 112, A10202. http://dx.doi.org/ 10.1029/2007JA012275.

Bunce, E.J. et al., 2008. Origin of Saturn's aurora: Simultaneous observations by Cassini and the Hubble Space Telescope. J. Geophys. Res. 113, A09209. http:// dx.doi.org/10.1029/2008JA013257.

Bunce, E.J. et al., 2014. Cassini nightside observations of the oscillatory motion of Saturn's northern auroral oval. J. Geophys. Res.. http://dx.doi.org/10.1002 2013JA019527.

Carbary, J.F., 2012. The morphology of Saturn's ultraviolet aurora. J. Geophys. Res. 117, A06210. http://dx.doi.org/10.1029/2012JA017670.

Carlson, C.W. et al., 1998. FAST observations in the downward auroral current regions: Energetic upgoing electron beams, parallel potential drops, and ion heating. Geophys. Res. Lett. 25, 2017-2020. http://dx.doi.org/10.1029/ 98GL00851.

Delamere, P.A., Wilson, R.J., Eriksson, S., Bagenal, F., 2013. Magnetic signatures of Kelvin-Helmholtz vortices on Saturn's magnetopause: Global survey. J. Geophys. Res.: Space Phys. 118, 393-404. http://dx.doi.org/10.1029/2012JA018197.

Dougherty, M.K. et al., 2004. The Cassini magnetic field investigation. Space Sci. Rev. $114,331-383$.

Dougherty, M.K. et al., 2005. Cassini magnetometer observations during Saturn orbit insertion. Science 307, 1266-1270. http://dx.doi.org/10.1126/SCIENCE.1106098.
Esposito et al., 2004. The Cassini ultraviolet imaging spectrograph investigation. Space Sci. Rev. 114, 299-361.

Gérard, J.-C., Bunce, E.J., Grodent, D., Cowley, S.W.H., Clarke, J.T., Badman, S.V., 2005. Signature of Saturn's auroral cusp: Simultaneous Hubble Space Telescope FUV observations and upstream solar wind monitoring. J. Geophys. Res. 110, A11201. http://dx.doi.org/10.1029/2005JA011094.

Gurnett, D.A. et al., 2004. The Cassini radio and plasma wave investigation. Space Sci. Rev. 114, 395-463.

Jasinski, J.M. et al., 2014. Cusp observation at Saturn's high-latitude magnetosphere by the Cassini spacecraft. Geophys. Res. Lett. 41, 1382-1388. http://dx.doi.org/ 10.1002/L059319.

Jinks, S.L. et al., 2014. Cassini multi-instrument assessment of Saturn's polar cap boundary. J. Geophys. Res. Space Phys. 119. http://dx.doi.org/10.1002/ 2014JA020367.

Klumpar, D.M., 1990. Near equatorial signatures of dynamic auroral processes. In: Physics of Space Plasmas. Sci. Publ., Cambridge, Mass, pp. 265-276.

Krimigis, S.M. et al., 2004. Magnetospheric imaging instrument (MIMI) on the Cassini mission to Saturn/Titan. Space Sci. Rev. 114, 233-329.

Marklund, G.T. et al., 2001. Temporal evolution of the electric field accelerating electrons away from the auroral ionosphere. Nature 414, 724-727. http:// dx.doi.org/10.1038/414724a.

Masters, A. et al., 2010. Cassini observations of a Kelvin-Helmholtz instability vortex in Saturn's outer magnetosphere. J. Geophys. Res. 115, A07225. http:// dx.doi.org/10.1029/A015351.

Meredith, C.J. et al., 2014. Saturn's dayside ultraviolet auroras: Evidence for morphological dependence on the direction of the upstream interplanetary magnetic field. J. Geophys. Res.: Space Phys. 119, 1994-2008. http://dx.doi.org/ 10.1002/2013JA019598.

Mitchell, D.G. et al., 2009a. Ion conics and electron beams associated with auroral processes on Saturn. J. Geophys. Res. 114, A02212. http://dx.doi.org/10.1029/ 2008JA013621.

Mitchell, D.G. et al., 2009b. Recurrent energization of plasma in the midnight-todawn quadrant of Saturn's magnetosphere, and its relationship to auroral UV and radio emissions. Planet. Space Sci. 57, 1732-1742. http://dx.doi.org/ 10.1016/j.pss.2009.04.002.

Mitchell, D.G. et al., 2014. Injection, interchange and reconnection. In: Keiling, A. Jackman, C.M., Delamere, P.A. (Eds.), Magnetotails in the Solar System, AGU Geophysical Monograph 207. John Wiley \& Sons Inc., pp. 327-344.

Nichols, J.D., Cowley, S.W.H., Lamy, L., 2010. Dawn-dusk oscillation of Saturn's conjugate auroral ovals. Geophys. Res. Lett. 37, L24102. http://dx.doi.org/ 10.1029/2010GL045818.

Nichols, J.D. et al., 2014. Dynamic auroral storms on Saturn as observed by the Hubble Space Telescope. Geophys. Res. Lett. 41, 3323-3330. http://dx.doi.org/ 10.1002/2014GL060186.

Radioti, A. et al., 2011. Bifurcations of the main auroral ring at Saturn: Ionospheric signatures of consecutive reconnection events at the magnetopause. J. Geophys. Res. 116, A11209. http://dx.doi.org/10.1029/2011JA016661.

Radioti, A. et al., 2013. Auroral signatures of multiple magnetopause reconnection at Saturn. Geophys. Res. Lett. 40, 4498-4502. http://dx.doi.org/10.1002/ grl.50889.

Talboys, D.L., Bunce, E.J., Cowley, S.W.H., Arridge, C.S., Coates, A.J., Dougherty, M.K., 2011. Statistical characteristics of field-aligned currents in Saturn's nightside magnetosphere. J. Geophys. Res. 116, A06220. http://dx.doi.org/10.1029/ 2010JA016102.

Thomsen, M.F., Reisenfeld, D.B., Delapp, D.M., Tokar, R.L., Young, D.T., Crary, F.J., Sittler, E.C., McGraw, M.A., Williams, J.D., 2010. Survey of ion plasma parameters in Saturn's magnetosphere. J. Geophys. Res. 115, A10220. http:// dx.doi.org/10.1029/2010JA015267.

Wilson, R.J., Delamere, P.A., Bagenal, F., Masters, A., 2012. Kelvin-Helmholtz instability at Saturn's magnetopause: Cassini ion data analysis. J. Geophys. Res.: Space Phys. 117, A03212. http://dx.doi.org/10.1029/2011JA016723. 